\title{
Humanização da assistência de enfermagem ao idoso na Atenção Básica: revisão integrativa
}

\author{
Humanization of nursing care for the elderly in Primary Care: an integrative review \\ Humanización de la atención de enfermería al anciano en Atención Primaria: una revisión \\ integradora
}

Recebido: 02/08/2021 | Revisado: 07/08/2021 | Aceito: 10/08/2021 | Publicado: 14/08/2021

Jeruzia Pinheiro Torres

ORCID: https://orcid.org/0000-0001-9551-0449

Centro Universitário Vale do Salgado, Brasil

E-mail: jeruziatorres@gmail.com

Rafael Bezerra Duarte

ORCID: https://orcid.org/0000-0002-2280-0864

Centro Universitário Vale do Salgado, Brasil

Universidade Estadual do Ceará, Brasil

E-mail: rafaelduarte@univs.edu.br

Roberta Peixoto Vieira

ORCID: https://orcid.org/0000-0003-0640-1772

Centro Universitário Vale do Salgado, Brasil E-mail: robertapeixoto@univs.edu.br

Clélia Patrícia da Silva Limeira

ORCID: https://orcid.org/0000-0001-5359-789X

Centro Universitário Vale do Salgado, Brasil

E-mail: clelia@univs.edu.br

Cidianna Emanuelly Melo do Nascimento

ORCID: https://orcid.org/0000-0001-5477-4413

Universidade Estadual do Ceará, Brasil

E-mail: profa.cidianna.melo@gmail.com

Carla Barbosa Brandão

ORCID: https://orcid.org/0000-0002-6608-488X

Universidade Estadual do Ceará, Brasil

E-mail: mailto:brandao.barbosa8214@gmail.com

Samir Gabriel Vasconcelos Azevedo

ORCID: https://orcid.org/0000-0003-1005-5980 Universidade Estadual do Ceará, Brasil

E-mail: samirueva@gmail.com

Daniele Keuly Martins da Silva

ORCID: https://orcid.org/0000-0002-5026-5176 Universidade Estadual do Ceará, Brasil E-mail: danikeuly@gmail.com

Kerma Márcia de Freitas

ORCID: https://orcid.org/0000-0003-3021-4758

Centro Universitário Vale do Salgado, Brasil

E-mail: kermamarcia@gmail.com

Maria Rocineide Ferreira da Silva

ORCID: https://orcid.org/0000-0002-6086-6901 Universidade Estadual do Ceará, Brasil

E-mail: rocineide.ferreira@ uece.br

\begin{abstract}
Resumo
Atualmente o processo de envelhecimento é compreendido como um desafio para a saúde pública mundial, visto o acentuado aumento da população idosa. Objetivou-se analisar a produção científica acerca da humanização da assistência de enfermagem ao idoso no contexto da Atenção Básica. Trata-se de uma revisão integrativa, compreendendo estudos nacionais publicados entre 2011 e 2020, no portal da Biblioteca Virtual de Saúde. A busca e seleção dos artigos ocorreu no período de maio a junho de 2021, através do cruzamento de descritores e uso do operador booleano: "Assistência de enfermagem" and "Idoso" and "Humanização". Na busca, pode-se obter um total de 160 artigos, após aplicação dos filtros restaram 68 para análise. Posteriormente leitura, análise e aplicação dos critérios de inclusão e exclusão sob os 68 estudos, restaram para compor a amostra 18 artigos. Depois da análise dos estudos, surgiram duas categorias: 1 - Assistência humanizada da enfermagem à pessoa idosa no âmbito da Atenção Básica e; 2 - Principais obstáculos enfrentados pelos enfermeiros para ofertar assistência ao idoso de forma
\end{abstract}


humanizada na Atenção Básica. Frente os resultados obtidos, pode-se evidenciar a importância da assistência humaniza a pessoa idosa, assim como, a existência de dificuldades que ainda impedem a oferta dessa assistência pelos profissionais de enfermagem. Portanto, se faz necessário a efetivação das políticas públicas para essa população, com vista a garantir o cuidado humanizado, vislumbrando um envelhecimento saudável e de qualidade, além de capacitação dos profissionais na área da geriatria e gerontologia.

Palavras-chave: Assistência de enfermagem; Idoso; Humanização da assistência.

\begin{abstract}
Currently, the aging process is understood as a challenge for public health worldwide, given the sharp increase in the elderly population. The objective was to analyze the scientific production about the humanization of nursing care for the elderly in the context of Primary Care. This is an integrative review, comprising national studies published between 2011 and 2020, on the Virtual Health Library portal. The search and selection of articles took place from May to June 2021, by crossing descriptors and using the operator Boolean: "Assistência de Enfermagem" and "idoso" and "humanização". In the search, a total of 160 articles can be obtained, after applying the filters, 68 remained for analysis. After reading, analyzing and applying the inclusion and exclusion criteria under the 68 studies, 18 articles remained to compose the sample. After analyzing the studies, two categories emerged: 1 - Humanized nursing care for the elderly in the context of Primary Care and; 2 - Main obstacles faced by nurses to provide care for the elderly in a humane way in Primary Care. Based on the results obtained, the importance of care can be seen to humanize the elderly, as well as the existence of difficulties that still prevent nursing professionals from offering this assistance. Therefore, it is necessary to implement public policies for this population, in order to ensure humanized care, envisioning healthy and quality aging, in addition to training professionals in the area of geriatrics and gerontology.
\end{abstract}

Keywords: Nursing care; Aged; Humanization of assistance.

\title{
Resumen
}

Actualmente, el proceso de envejecimiento se entiende como un desafío para la salud pública a nivel mundial, dado el fuerte aumento de la población anciana. El objetivo fue analizar la producción científica sobre la humanización de la atención de enfermería al adulto mayor en el contexto de la Atención Primaria. Se trata de una revisión integradora, que comprende los estudios nacionales publicados entre 2011 y 2020, en el portal de la Biblioteca Virtual en Salud. La búsqueda y selección de artículos se realizó de mayo a junio de 2021, cruzando descriptores y utilizando el operador booleano: "Assistência de Enfermagem" and "idoso" and "humanização". En la búsqueda se pueden obtener un total de 160 artículos, luego de aplicar los filtros, 68 quedaron para análisis. Después de leer, analizar y aplicar los criterios de inclusión y exclusión de los 68 estudios, quedaron 18 artículos para componer la muestra. Tras analizar los estudios, surgieron dos categorías: 1 - Atención de enfermería humanizada al anciano en el contexto de Atención Primaria y; 2 - Principales obstáculos que enfrentan las enfermeras para brindar una atención humana al adulto mayor en Atención Primaria. A partir de los resultados obtenidos, se aprecia la importancia del cuidado para humanizar al anciano, así como la existencia de dificultades que aún impiden a los profesionales de enfermería ofrecer esta asistencia. Por ello, es necesario implementar políticas públicas para esta población, con el fin de asegurar una atención humanizada, vislumbrando un envejecimiento saludable y de calidad, además de formar profesionales en el área de geriatría y gerontología.

Palabras clave: Atención de enfermería; Anciano; Humanización de la atención.

\section{Introdução}

O envelhecimento é um processo sequencial, individual e irreversível. Trata-se de uma etapa da vida marcada por progressivas transformações, como, biológica, física, psicológica e sociocultural. Estas apresentam características marcantes do processo de envelhecimento, agregando ao idoso diferentes posturas e formas de ser, bem como de se comportar. Este processo ainda pode ser descrito de duas maneiras, a senescência e senilidade, sendo a primeira delas um processo natural, fisiológico e esperado para aquela idade, e a segunda se caracteriza por um processo patológico gerando modificações causadas por distúrbios ligados ao envelhecimento (Freitas, 2017).

Nos dias atuais o processo de envelhecimento é compreendido como um desafio para a saúde pública mundial, visto que, estão acontecendo significativas mudanças nas composições etárias dos países em desenvolvimento, compreendendo dessa forma um acentuado aumento da população idosa (Tavares et al., 2017). O Brasil passa por uma significativa transformação em sua pirâmide etária, comprovando-se pelo gradativo aumento da sua população. Este evento se desencadeou devido as mudanças demográficas e epidemiológicas, assim como, por consequência da diminuição das taxas de fecundidade e mortalidade, aumentando assim, a expectativa de vida da população (Veras \& Oliveira, 2018). 
Compreende-se o envelhecimento como um processo cumulativo e, segundo dados da Organização Mundial de Saúde (OMS), nos próximos anos a população de idosos (indivíduos com idade igual ou superior a 60 anos) em todo o mundo vai passar dos atuais 841 milhões para 2 bilhões até 2050. No Brasil, dados do Instituto Brasileiro de Geografia e Estatística (IBGE), alusivos ao ano de 2018, assinalam que a população idosa alcançará os 29 milhões e a expectativa é que, até 2060, este número eleve-se para 73 milhões, representando assim um aumento de 160\%. Em 2032, o Brasil será considerado um país velho, quando 32,5 milhões dos mais de 226 milhões de brasileiros apresentarão idade de 65 anos ou mais. Ainda, de acordo com a OMS, acrescenta-se que até o ano de 2025, o Brasil será o sexto país do mundo em número de pessoas idosas (IBGE, 2018).

Diante desse panorama de transição demográfica, destacamos a importância da qualidade de vida dos idosos, assim como, a atual situação de saúde em que a mesma se encontra ou apresentará no futuro, pois, para muitos o processo de envelhecimento é acompanhado por Doenças Crônicas não Transmissíveis (DCNT), limitações ambientais, socioculturais e socioeconômicas, perda da independência e autonomia, decaimento da capacidade cognitiva, entre outros fatores. Estes por sua vez, acarretam sérios prejuízos a qualidade de vida, assim como, podem estar associados à limitação da capacidade funcional dos idosos, interferindo diretamente na realização de suas atividades de vida diária, levando-os a necessitarem de assistência de qualidade e humanizada (Nunes et al., 2017; Moreia, 2017).

Portanto, é imprescindível a assistência da enfermagem na terceira idade, pois sabe-se que são usuários que necessitam de uma atenção em saúde maior que as demais faixas etárias. Visto que, o enfermeiro é um dos responsáveis por promover o cuidado, seu papel em ofertar a assistência de forma humana se torna fundamental. Deste modo, os cuidados de enfermagem a pessoa idosa são indispensáveis para a melhoria do estado de saúde, seja eles para a manutenção ou a obtenção de estilos de vida saudáveis, ou por situação de doença que acarretam a aquisição do bem-estar ou à promoção da independência (Vieira \& Almeida, 2020).

Visto que existe uma enorme necessidade de cuidados nessa fase da vida, evidências mostram a importância de trabalhar a assistência de forma humanizada, tendo em vista atender as necessidades básicas e buscar tratamento holístico para o processo de saúde e doença. Neste sentido, humanizar em saúde é atender as necessidades do outro com responsabilidade, e entender suas necessidades, reconhecendo seus direitos, pois se trata de seres que possuem sentimentos, famílias e histórias. Nas ações de assistência se faz necessário entender as complexidades e necessidades básicas da pessoa idosa, pois humanizar é ofertar cuidados integrais ao indivíduo (Silva \& Silva, 2017; Brasil, 2010).

Destarte, o principal desafio da enfermagem em relação a humanização e saúde do idoso, é inseri-lo dentro do processo de promoção a saúde, fazendo com que o idoso entenda e tenha acesso a informações a respeito das políticas de forma clara e objetiva. Portanto, o enfermeiro precisa atuar na identificação e atentar-se em necessidades individuais desses indivíduos, mostrando-as aos cuidadores e familiares, de forma a prestar um melhor cuidado prezando pela autonomia, que muitas vezes não são trabalhadas para que esta população assume seu papel perante a sociedade (Silva \& Borges, 2014).

Diante da problemática apresentada, percebe-se como a humanização precisa ser trabalhada pelos enfermeiros durante a assistência prestada ao idoso, sobretudo, no contexto da Atenção Básica, uma vez que se trata da porta de entrada do usuário ao Sistema Único de Saúde (SUS), e promotora do cuidado. Logo, faz-se necessário à realização de pesquisas sobre a humanização da assistência de enfermagem a população idosa, tendo em vista que, infelizmente ainda se vê durante alguns atendimentos de enfermagem a ausência de humanização em diversas áreas da assistência. Assim, surge o questionamento: O que as publicações trazem acerca da humanização da assistência de enfermagem ao idoso no contexto da Atenção Básica?

Deste modo, o presente estudo torna-se relevante, pois proporcionará o aumentar de conhecimentos sobre a temática, bem como nos trará informações pertinentes que poderão despertar no meio acadêmico o interesse por novas pesquisas, assim 
como, servirá como fonte de pesquisa. Ademais, com os resultados obtidos, poderá ser utilizado para o planejamento de novas ações estratégicas relacionadas ao cuidado humanizado, visando uma melhoria na qualidade de vida da população idosa.

Destarte, este artigo tem como objetivo analisar a produção científica acerca da humanização da assistência de enfermagem ao idoso no contexto da Atenção Básica.

\section{Metodologia}

Trata-se de uma revisão integrativa, alusivo à produção científica sobre a humanização da assistência de enfermagem ao idoso no contexto da Atenção Básica. De acordo com Pompeo, Rossi e Galvão (2009), esse tipo de estudo é amplo, por sua vez, possibilita ao pesquisador um resumo e análise do conhecimento científico obtido sobre um assunto já pesquisado.

Os autores ainda destacam que, os estudos que são incluídos neste tipo de pesquisa são analisados sistematicamente conforme seus objetivos, materiais e métodos, fazendo com que o leitor analise o conhecimento pré-existente de uma temática pesquisado.

A presente revisão segue o modelo de Botelho, Cunha e Macedo (2011), o qual foram percorridas seis etapas. Estas etapas seguidas foram: identificação do tema e seleção da questão de pesquisa; estabelecimento dos critérios de inclusão e exclusão; identificação dos estudos pré-selecionados e selecionados; categorização dos estudos selecionados; análise e interpretação dos resultados; e, apresentação da revisão/síntese do conhecimento.

A partir da problemática levantada, a busca por artigos ocorreu durante o período de maio a junho de 2021, no portal da Biblioteca Virtual em Saúde (BVS). Justifica-se que a busca ocorreu em apenas uma base de dados porque a BVS abrange diversas revistas na área da saúde que publicam manuscritos que respondem à questão de pesquisa para formar a amostra desse artigo.

Para a busca dos artigos foi realizado o cruzamento de descritores e uso do operador booleano: "Assistência de enfermagem" and "Idoso" and "Humanização".

Para a seleção dos artigos, levou-se em consideração os seguintes critérios de inclusão artigos: Disponíveis eletronicamente; Publicados na íntegra; Escritos na língua portuguesa; Formato - Artigos científicos (pesquisas qualitativas, quantitativas, quali/quantitativas, relatos de experiências) e; Publicados no período de 2011 a 2020.

Foram excluídos artigos: Revisão; Repetidos; e Artigos que estiverem fora da temática em estudo e/ou por não atenderem aos critérios de elegibilidade.

O processo de triagem dos artigos deu-se por meio da leitura dos títulos e resumos dos artigos surgidos a partir da equação de busca seguido por uma leitura na íntegra antes de selecioná-los para a coleta de dados.

Após a busca e análise dos estudos na BVS, por meio do uso dos descritores selecionados, pode-se alcançar a seguinte amostragem: 
Figura 1 - Fluxograma de seleção dos estudos que compuseram a revisão. Icó, Ceará, Brasil, 2021.

160 Artigos encontrados no portal a BVS na primeira busca

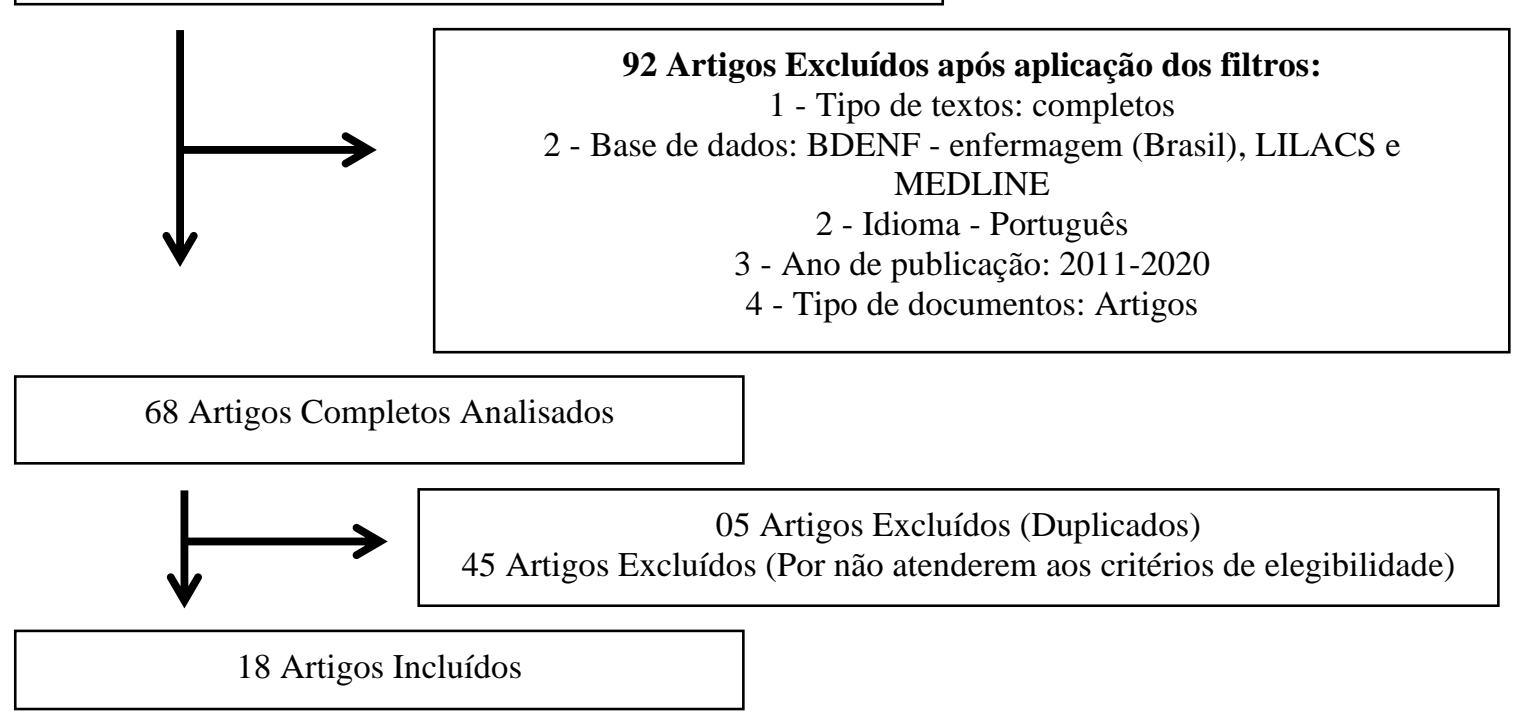

Fonte: Resultados da pesquisa.

Para a organização dos dados, bem como a análise, foi feita leitura e releitura de todo o material selecionado. Os principais dados dos estudos selecionados foram extraídos em um formulário de coleta de dados, adaptado para a condução da leitura e extração dos dados. O presente formulário foi adaptado do modelo de instrumento de coleta validado por Ursi (2005), o qual apresentou em sua estrutura, ano de publicação, autores, título do artigo, e os objetivos propostos pelas pesquisas.

Depois do mapeamento e organização dos estudos selecionados, os dados foram analisados através da análise de conteúdo proposta por Bardin (2011), o qual descreve que esse tipo de análise precisa ocorrer por meio de três fases, sendo a primeira a pré-análise do material, a segunda a exploração desse material e por último, o tratamento dos resultados, onde se faz a inferência e a interpretação dos mesmos.

Ainda, as fases de análise dos artigos e de interpretação dos resultados foram realizadas de forma impessoal, revisada em pares, delineada de forma criteriosa, buscando deste modo uma melhor compreensão dos resultados, discutindo a associação com os aspectos relacionados a resposta da pergunta norteadora desta pesquisa.

\section{Resultados e Discussão}

Após a busca dos estudos encontrados na BVS, foram selecionados para compor essa pesquisa 18 artigos, os quais foram analisados e utilizados para compor a discussão dessa pesquisa. No quadro abaixo encontra-se a síntese dos artigos, de acordo com ano de publicação, autores, título do artigo e os objetivos propostos pelas pesquisas, os mesmos versam acerca da assistência de enfermagem ao idoso no âmbito da Atenção Básica. 
Quadro 1 - Perfil da amostra selecionada. Icó, Ceará, Brasil, 2021.

\begin{tabular}{|c|c|c|c|}
\hline $\begin{array}{c}\text { Ano de } \\
\text { publicação }\end{array}$ & Autores & Título do artigo & Objetivo proposto \\
\hline 2011 & $\begin{array}{l}\text { ROCHA, F. C. V. et } \\
\text { al. }\end{array}$ & $\begin{array}{l}\text { O cuidado do enfermeiro ao idoso na } \\
\text { estratégia saúde da família. }\end{array}$ & $\begin{array}{l}\text { Descrever e discutir o cuidado do enfermeiro ao idoso na } \\
\text { estratégia saúde da família (ESF), bem como analisar os } \\
\text { aspectos que facilitam ou dificultam este cuidado. }\end{array}$ \\
\hline 2011 & $\begin{array}{l}\text { LINCK, C. L.; } \\
\text { CROSSETTI, M. G. } \\
\text { O. }\end{array}$ & $\begin{array}{l}\text { FRAGILIDADE NO IDOSO: o que vem } \\
\text { sendo produzido pela enfermagem. }\end{array}$ & $\begin{array}{l}\text { Este trabalho visa identificar a produção científica } \\
\text { desenvolvida pela enfermagem frente à fragilidade no } \\
\text { idoso. }\end{array}$ \\
\hline 2012 & $\begin{array}{l}\text { CAMPOS, C. N. A. } \\
\text { et al. }\end{array}$ & $\begin{array}{l}\text { Reinventando práticas de enfermagem na } \\
\text { educação em saúde: teatro com idosos. }\end{array}$ & $\begin{array}{l}\text { O estudo apresenta como objetivo elaborar uma } \\
\text { intervenção de enfermagem em educação em saúde, com } \\
\text { enfoque na promoção à saúde de um grupo de idosos, } \\
\text { utilizando como ferramenta as artes cênicas. }\end{array}$ \\
\hline 2013 & $\begin{array}{l}\text { SOUSA L; RIBEIRO } \\
\text { A. P. }\end{array}$ & $\begin{array}{l}\text { Prestar cuidados de enfermagem a pessoas } \\
\text { idosas: experiências e impactos. }\end{array}$ & $\begin{array}{l}\text { Os idosos constituem o grupo populacional que } \\
\text { atualmente mais recorre aos serviços de saúde e, também, } \\
\text { aos cuidados de enfermagem. Este estudo exploratório } \\
\text { examina as experiências dos enfermeiros na prestação de } \\
\text { cuidados a pessoas idosas e identifica seus impactos nos } \\
\text { enfermeiros. }\end{array}$ \\
\hline 2013 & $\begin{array}{l}\text { OLIVEIRA, J. M. M. } \\
\text { et al. }\end{array}$ & $\begin{array}{l}\text { O cuidado de enfermagem na visita } \\
\text { domiciliar gerontológica: uma perspectiva } \\
\text { humanística. }\end{array}$ & $\begin{array}{l}\text { Descrever uma experiência de ensino e aprendizagem em } \\
\text { assistência domiciliar vivenciada por discentes de } \\
\text { enfermagem, com enfoque na teoria de Paterson e Zderad. }\end{array}$ \\
\hline 2013 & SANTOS, R. S. et al. & $\begin{array}{l}\text { A assistência domiciliar ao idoso na } \\
\text { perspectiva dos enfermeiros. }\end{array}$ & $\begin{array}{l}\text { Analisar a atenção domiciliar à pessoa idosa sob a } \\
\text { perspectiva dos profissionais de enfermagem. }\end{array}$ \\
\hline 2014 & $\begin{array}{l}\text { ALBERTI, G. F.; } \\
\text { ESPÍNDOLA, R. B.; } \\
\text { CARVALHO, S. O. } \\
\text { R. M. }\end{array}$ & $\begin{array}{l}\text { Abordagem ao idoso na estratégia de } \\
\text { saúde da família e as implicações para a } \\
\text { atuação do enfermeiro. }\end{array}$ & $\begin{array}{l}\text { Identificar as ações de cuidado do enfermeiro da Atenção } \\
\text { Primária à Saúde em relação ao idoso. }\end{array}$ \\
\hline 2014 & $\begin{array}{l}\text { OLIVEIRA, A. M. } \\
\text { S.; MENEZES, T. M. } \\
\text { O. }\end{array}$ & $\begin{array}{l}\text { A enfermeira no cuidado ao idoso na } \\
\text { estratégia saúde da família: sentidos do } \\
\text { vivido. }\end{array}$ & $\begin{array}{l}\text { Este estudo objetivou compreender os sentidos do vivido } \\
\text { da enfermeira no cuidado à pessoa idosa na estratégia } \\
\text { saúde da família. }\end{array}$ \\
\hline 2014 & SILVA P. L. N. et al. & $\begin{array}{l}\text { Assistência psicológica e de enfermagem } \\
\text { ao idoso na atenção primária. }\end{array}$ & $\begin{array}{l}\text { Descrever o processo de assistência de enfermagem e } \\
\text { também psicológica ao idoso na Atenção Primária. }\end{array}$ \\
\hline 2015 & $\begin{array}{l}\text { BRITO, R. F. S. L. } \\
\text { V. et al. }\end{array}$ & $\begin{array}{l}\text { O idoso na estratégia saúde da família: } \\
\text { atuação do enfermeiro durante o } \\
\text { envelhecimento ativo. }\end{array}$ & $\begin{array}{l}\text { O objetivo do estudo foi analisar a atuação do enfermeiro } \\
\text { da Estratégia de Saúde da Família (ESF) para efetivação } \\
\text { da assistência do enfermeiro durante o processo de } \\
\text { envelhecimento ativo. }\end{array}$ \\
\hline 2015 & $\begin{array}{l}\text { RESENDE, J. O. et } \\
\text { al. }\end{array}$ & $\begin{array}{l}\text { Assistência do enfermeiro ao idoso na } \\
\text { estratégia saúde da família. }\end{array}$ & $\begin{array}{l}\text { O objetivo deste estudo foi identificar a assistência } \\
\text { prestada pelo Enfermeiro aos idosos nas Estratégias Saúde } \\
\text { da Família no município de Divinópolis Minas Gerais, } \\
\text { levantar o perfil dos enfermeiros pesquisados e } \\
\text { caracterizar sua atuação na Saúde do idoso. }\end{array}$ \\
\hline 2016 & $\begin{array}{l}\text { NOGUEIRA, I. S. et } \\
\text { al. }\end{array}$ & $\begin{array}{l}\text { Intervenção domiciliar como ferramenta } \\
\text { para o cuidado de enfermagem: avaliação } \\
\text { da satisfação de idosos. }\end{array}$ & $\begin{array}{l}\text { Avaliar os resultados de intervenções domiciliares de } \\
\text { enfermagem na perspectiva da satisfação de idosos. }\end{array}$ \\
\hline 2016 & LEITE, B. S. et al. & $\begin{array}{l}\text { Consultas de enfermagem aos idosos em } \\
\text { assistência básica no intercâmbio } \\
\text { estudantil internacional: relato de } \\
\text { experiência. }\end{array}$ & $\begin{array}{l}\text { Descrever a experiência discente sobre as consultas de } \\
\text { enfermagem à população idosa durante o intercâmbio } \\
\text { estudantil internacional. }\end{array}$ \\
\hline 2017 & $\begin{array}{l}\text { EMILIANO, M. S. et } \\
\text { al. }\end{array}$ & $\begin{array}{l}\text { A percepção da consulta de enfermagem } \\
\text { por idosos e seus cuidadores. }\end{array}$ & $\begin{array}{l}\text { Conhecer a percepção dos idosos com Alzheimer e seus } \\
\text { cuidadores consultados no programa de extensão } \\
\text { Enfermagem na Atenção à Saúde do Idoso e seu cuidador } \\
\text { a respeito da consulta de enfermagem. }\end{array}$ \\
\hline 2018 & $\begin{array}{l}\text { SAMPAIO, S. N. et } \\
\text { al. }\end{array}$ & $\begin{array}{l}\text { Visão da pessoa idosa sobre o atendimento } \\
\text { do enfermeiro da Atenção Básica. }\end{array}$ & $\begin{array}{l}\text { Descrever a visão da pessoa idosa a respeito do } \\
\text { atendimento do enfermeiro na atenção básica de saúde. }\end{array}$ \\
\hline
\end{tabular}




\begin{tabular}{|l|l|l|l|}
\hline 2019 & $\begin{array}{l}\text { COSTA, J. B.; } \\
\text { FURTADO, L. G. S; } \\
\text { GIRARD, C. C. P. }\end{array}$ & $\begin{array}{l}\text { Saberes e práticas do enfermeiro na } \\
\text { consulta com o idoso na estratégia saúde } \\
\text { da família }\end{array}$ & $\begin{array}{l}\text { Conhecer os saberes e as práticas do enfermeiro frente ao } \\
\text { cuidado à saúde da pessoa idosa na Estratégia Saúde da } \\
\text { Família. }\end{array}$ \\
\hline 2020 & $\begin{array}{l}\text { MENEZES, T. M. O. } \\
\text { et al. }\end{array}$ & $\begin{array}{l}\text { Acolhimento e cuidado da enfermeira na } \\
\text { estratégia saúde da família: percepções da } \\
\text { pessoa idosa. }\end{array}$ & $\begin{array}{l}\text { Analisar a percepção da pessoa idosa sobre o acolhimento } \\
\text { e cuidado da enfermeira na Estratégia Saúde da Família. }\end{array}$ \\
\hline $\begin{array}{l}\text { FREITAS, M. A.; } \\
\text { ALVAREZ, A. M. }\end{array}$ & $\begin{array}{l}\text { Melhores práticas de enfermagem na na da pessoa idosa. } \\
\text { sauude da melhores práticas, as } \\
\text { experiências de busca por conhecimento e utilização da } \\
\text { experiência profissional dos enfermeiros no cuidado da } \\
\text { pessoa idosa na Atenção Primária à Saúde. }\end{array}$ \\
\hline
\end{tabular}

Fonte: Resultados da pesquisa.

A partir da leitura e análise dos artigos selecionados para compor esse estudo, pode-se agrupar os resultados por conteúdos parecidos, emergindo assim a construção de duas categorias.

\subsection{Categoria 1 - Assistência humanizada da enfermagem à pessoa idosa no âmbito da Atenção Básica}

A assistência humanizada da Enfermagem ao idoso é de fácil compreensão. A enfermagem trabalha através da Sistematização da Assistência de Enfermagem (SAE), uma prática que torna possível uma assistência humanizada, pois a mesma torna o processo de trabalho da enfermagem menos mecânico. Dessa forma, o profissional consegue agir de acordo com a priorização do cuidado prestado, individualizando o mesmo e estendendo-o para o idoso e família, e até mesmo para os cuidadores (Silva, 2019).

Para humanizar os cuidados na saúde da pessoa idosa é preciso estabelecer um vínculo de confiança e amizade com o idoso e muitas vezes com a família deste, conhecer as necessidades e limitações de cada paciente, ser afetivo, saber se comunicar, ter empatia e profissionalismo, ou seja, é saber falar e saber ouvir até mesmo os silêncios, é se doar de coração, assim, oferecer um atendimento ao idoso composto por ações éticas e respeitosas (Zoboli \& Schveitzer, 2013).

Diante do exposto, a presente categoria apresentará os principais achados acerca da assistência prestada pela enfermagem à pessoa idosa no âmbito da Atenção Básica, com foco na assistência humanizada.

Segundo Nogueira et al. (2016) para se ofertar uma assistência humanizada a população idosa se faz necessário o fortalecimento do vínculo afetivo, assim como a criação de parcerias entre os envolvidos no cuidado, através da construção coletiva das intervenções terapêuticas tendo como foco as necessidades apresentadas. Em sua pesquisa os autores supracitados revelam ainda que, o vínculo verbalizado pode possibilitar uma melhor interação entre enfermeiros, familiares e idosos, proporcionando assim, o estabelecimento de forte aliança terapêutica, fato esse que colabora para as orientações individuais e pactuação do autocuidado entre a população idosa.

Por ser a Atenção Básica o primeiro contato do paciente aos serviços de saúde, torna-se um ambiente onde se pode ter uma melhor criação de vínculo entre profissionais e pacientes, principalmente os pacientes idosos, considerados o público que mais procura por serviços de saúde. Logo esse vínculo auxilia para a prestação de uma assistência humanizada, na qual deve consistir na integralidade, longitudinalidade, orientação familiar e comunitária (Alberti, Espíndola \& Carvalho, 2014).

No cotidiano profissional e assistencial dos enfermeiros dentro da Atenção Básica, se faz necessário um olhar diferenciado ao paciente idoso. É preciso dar prioridade ao idoso, assim como, atenção, ouvir suas principais queixas, e acima de tudo respeito. Assim sendo, no estudo de Rocha et al. (2011) pode-se observar que, o acolhimento dos idosos na Atenção Básica se mostrou como elemento positivo frente a assistência prestada, evidenciando-se como uma forma de superação de deficiências e dificuldades existentes, sendo possível garantir certo grau de resolutividade e satisfação da população idosa. Os achados desse estudo também apontaram que a afetividade do enfermeiro em relação ao idoso foi outro modo de cuidado demonstrado e expresso de forma humanizada. 
Oliveira e Menezes (2014), destacam em sua pesquisa que a assistência humanizada de enfermagem parte também da solicitude, forma de como os envolvidos se relacionam, forma de cuidar um do outro. Logos, isso só é possível mediante uma relação envolvente, a qual se caracteriza por meio da consideração de um para com o outro.

$\mathrm{Na}$ pesquisa de Brito et al. (2015) os dados evidenciam a importância da assistência do enfermeiro no acompanhamento ao idoso, pois sua presença durante o processo de cuidado torna o idoso mais confiante na equipe da Atenção Básica, além de despertar sua curiosidade e interesse. Os dados dessa pesquisa também apontam que a aproximação pode trazer vantagens à assistência do enfermeiro, uma vez que, ao lhe oportunizar uma observação mais detalhada e continuada, ajudara na prevenção e identificação de patologias ou outro problema que os aflijam a saúde e vida da pessoa idosa.

Silva et al. (2014) em sua pesquisa destacam que os profissionais enfermeiros ocupam papel fundamental na atenção à saúde da população idosa, e que dentro da Atenção Básica esses profissionais são os que tem o primeiro contato com esse público quando os mesmos apresentam algum problema de saúde. Os autores também destacam a importância de estabelecer o vínculo entre a família e a equipe, além da importância de prestar uma assistência em conformidade aos princípios do SUS destacando a longitudinalidade e a integralidade do cuidado.

No estudo de Oliveira et al. (2013) também pode-se identificar que no cotidiano da prática de enfermagem dentro da Atenção Básica, a integralidade do cuidado é de suma importância para que se tenha uma assistência mais humanizada ao idoso. Para Costa, Furtado e Girard (2019), a consulta de enfermagem ao idoso é uma ferramenta de assistência de suma importância, pois a mesma visa a prevenção, tratamento e o acompanhamento do estado de saúde desse público.

No estudo de Linck e Crossetti (2011), evidenciou-se que o profissional enfermeiro tem papel ímpar no cuidado individual do idoso, assim como no suporte aos familiares e aos cuidadores dos idosos, tem como destaque os idosos frágeis. Santos et al. (2013) também apontam que para que se tenha uma assistência de qualidade e mais humanizada se faz necessária uma interação entre os profissionais, idoso e família. Já Campos et al. (2012), destacam em sua pesquisa que os profissionais de enfermagem precisam munir-se de novos saberes para ofertar uma assistência mais qualificada as necessidades da população idosa.

Para que se tenha uma assistência de qualidade e humanizada ao paciente idoso, Leite et al. (2016) destacam a importância do desenvolvimento das consultas de enfermagem, pois as mesmas podem favorecer a identificação das principais necessidades dos pacientes, além de possibilitar a elaboração de um plano de cuidados eficaz para a promoção e prevenção da saúde. Os autores também destacam em sua pesquisa que as consultas de enfermagem além de serem benéficas aos pacientes, trazem também benefícios aos profissionais que as realizam por meio do aperfeiçoamento do pensamento crítico e reflexivo, da capacidade de comunicação e percepção da linguagem verbal ou não verbal, assim como através do desenvolvimento das habilidades relacionadas ao processo de enfermagem, da promoção de cuidados, do estabelecimento de vínculo, da relação de confiança entre profissional e paciente, dentre outros inúmeros benefícios.

Emiliano et al. (2017) também apontam a importância que as consultas de enfermagem têm em relação a uma melhor assistência humanizada, uma vez que, no momento da consulta os profissionais podem além da prestação do cuidado, estabelecerem uma relação de confiança com os idosos, familiares e cuidadores, assim como, promover a orientação para o autocuidado.

Sampaio et al. (2018) destacam que para se ter uma assistência humanizado no cuidado ao idoso é indispensável um olhar direcionado, integral e sistematizado. É importante também compreendendo que esta assistência envolve o ambiente em que se encontra o idoso e seus aspectos culturais e socioeconômicos.

De acordo com Menezes et al. (2020) alguns fatores são fundamentais para o sucesso da assistência a pessoa idosa como por exemplo, a empatia com destaque na escuta qualificada e o cuidado no acolhimento e afetuosidade. Estes por sua 
vez, permitiram que equipe de enfermagem promova aproximação com confiança, proporcionando formação de vínculo e comunicação efetiva com a população idosa, favorecendo assim uma assistência mais humanizada.

Um dos estudos nos chamou a atenção, quando o mesmo revela que os membros da equipe multiprofissional da Atenção Básica entendem que o profissional enfermeiro é de suma importância quando se trata do cuidado, pois, é um profissional capaz de articular e integrar os saberes (conhecer, fazer, ser e conviver), possibilitando deste modo uma assistência com qualidade ao usuário e, consequentemente, a promoção de melhores práticas de cuidado (Freitas \& Alvarez, 2020).

Diante do exposto, fica notória que a função do profissional da enfermagem em relação ao idoso tem sido a de promover práticas e ações voltadas para a saúde, objetivando a melhoria da autonomia, independência, bem-estar e qualidade de vida deste público específico. Dessa forma o enfermeiro juntamente com uma equipe interdisciplinar tem condições de buscar meios para trabalhar de uma forma humanizada e prestar uma assistência integral e de qualidade para o público senil (Veras \& Oliveira, 2018).

Além disso, cabe mencionar a importância do papel de enfermagem em transmitir confiança, respeito e empatia ao prestar assistência ao paciente idoso. Também se sabe que para ofertar um cuidado humanizado, o profissional de enfermagem necessita compreender as necessidades e limitações de cada paciente e estabelecer um vínculo de confiança e amizade com o idoso, cuidadores e seus familiares.

A afetividade do enfermeiro no acolhimento aos idosos dentro da Estratégia de Saúde da Família pode propiciar uma assistência com resultado positivo, pois, essa oportunidade de orientações devido a reciproca confiança instalada é imprescindível para promover o envelhecimento ativo e saudável.

A humanização da assistência de enfermagem ao idoso fundamenta-se na agregação de atitudes como comunicação, dialogo, afeto familiar, confiança e empatia, criando assim melhores condições para a promoção da saúde e bem-estar dos pacientes. O cuidado ao idoso deve ser consolidado por meio de ações éticas, profissionais, humanas e respeitosas.

\subsection{Categoria 2 - Principais obstáculos enfrentados pelos enfermeiros para ofertar assistência ao idoso de forma humanizada na Atenção Básica}

Essa categoria evidencia os principais obstáculos que os profissionais enfermeiros enfrentam para oferecer assistência ao idoso de forma humanizada na Atenção Básica.

Segundo Rocha et al. (2011) a falta de capacitação profissional, carência de recursos materiais, assim como a falta de outros recursos humanos envolvidos no processo do cuidar, são os principais obstáculos que os profissionais de enfermagem encontram na Atenção Básica, dificultando assim a oferta de uma assistência ao idoso mais humanizada.

Nos estudos de Sampaio et al. (2018) e Sousa \& Ribeiro (2013), pode-se identificar que a falta de capacitação dos profissionais na área da geriatria e gerontologia são considerados obstáculos para ofertar uma assistência ao idoso de forma humanizada.

Evidenciou-se também em meio aos estudos que os problemas mais frequentes que os profissionais de enfermagem encontram para o atendimento aos idosos na Atenção Básica, são a falta de medicação e, problemas na infraestrutura das unidades (Brito et al., 2015).

Na pesquisa de Campos et al. (2012) identificou-se que um dos desafios dos profissionais da Atenção Básica é estabelecer a confiança dos idosos nos profissionais de saúde. Já na pesquisa de Resende et al. (2015) evidenciou-se que a população idosa recebe assistência e cuidados em suas necessidades como um usuário de qualquer faixa etária, mas não é assistido dentro das peculiaridades, se tornado um dos principais obstáculos encontrados para uma assistência mais humanizada. 
No estudo de Alberti, Espíndola e Carvalho (2014) pode-se evidenciar que o que dificulta os profissionais em muitos dos casos para ofertar uma assistência mais humanizada ao idoso na Atenção Básica é a ausência dos próprios idosos na unidade, muita das vezes os idosos só aparecem para pegar a medicação, não comparecem mensamente as consultas agendadas, sendo portando um dos obstáculos para a continuidade do cuidado.

Oliveira \& Menezes (2014) também destacam em sua pesquisa que um dos principais obstáculos que os profissionais de enfermagem encontram na Atenção Básica para ofertar uma assistência mais humanizada a população idosa e a falta de capacitação continuada em saúde da pessoa idosa. Os dados dessa pesquisa também apontam que os próprios profissionais reconhecem a importância da capacitação nesta área, e, consequentemente, no tipo de cuidado prestado a esses pacientes, tendo em vista a variedade de alterações decorrentes do processo de envelhecimento.

Sousa e Ribeiro (2013) descrevem em seu estudo que muitos profissionais ainda desconhecem os direitos dos idosos e agem com boa intenção, contudo sem informação e formação, o que pode afetar negativamente o cuidado e assistência à saúde da pessoa idosa. A falta de apoio e suporte familiar a pessoa idosa também é considero um dos obstáculos enfrentados pelos profissionais de enfermagem da Atenção Básica, frente a assistência humanizada (Oliveira et al., 2013).

Diante dos achados, evidencia-se a necessidade de melhoria na gestão dos serviços de saúde e na assistência à saúde da população idosa, pois, mesmo com avanços e melhorias, ainda se configura em cuidados superficiais e básicos.

Destarte, se faz necessário que os profissionais se atentem para o cumprimento efetivo das políticas públicas de Atenção à Saúde da Pessoa Idosa, assim como busquem se capacitar na área de geriatria e gerontologia para assim assistirem aos idosos de forma qualificada, integral e singular frente às suas reais necessidades.

\section{Considerações Finais}

A humanização no trabalho de enfermagem é uma necessidade atual, a qual exige que o profissional repense suas ações. Humanização não se trata apenas de outro tipo de cuidado, mas também englobam situações de respeito, apoio, diálogo e empatia, conceitos estes que precisam da efetividade na atuação do enfermeiro.

Deste modo, a humanização no serviço de enfermagem vai além do mero atendimento técnico, pois se preocupa com a palavra, com a comunicação, com a informação, com as necessidades do paciente. A promoção do cuidado e o tratamento são importantes, mas, é preciso, também, o amparo e o ato humanizado que perpassa as fragilidades, as limitações, as possibilidades e os anseios do paciente.

Percebe-se que o trabalho de enfermagem caminha gradativamente em direção a uma assistência humanizada e de qualidade, a qual vem avançando bastante no atendimento prestado aos pacientes, principalmente com a ajuda de tecnologias modernas e bem equipadas, que trouxeram muitos benefícios e agilidade ao campo da saúde, proporcionando a eficácia, bom tratamento, chance de cura e maior qualidade de vida.

Frente aos resultados obtidos nessa pesquisa, pode-se evidenciar que ainda há muito trabalho a ser feito no que se refere a assistência humanizada e cuidado a população idosa no contexto da Atenção Básica. Também, verificou-se que a assistência de enfermagem voltada ao paciente idoso tem enfrentado algumas dificuldades, as quais limitam sua atuação e uma boa assistência, como a falta de formação especializada, carga de trabalho pesada, ações governamentais para garantir o atendimento prioritário ao idoso não existem ou são ineficazes e outros obstáculos que impedem que os idosos recebam cuidados diferenciados e específicos.

Enfim, as evidências mostram, que os enfermeiros compreendem a necessidade de uma atenção mais humanizada e cuidadosa em relação à pessoa idosa, porém, as grandes demandas da profissão tornam a sua assistência mecanizada e tecnicista. 
Diante da importância do cuidado humanizado da enfermagem, é necessário que os profissionais de enfermagem, sobretudo, o enfermeiro resinifique a sua prática constantemente e alie a sua atuação técnica a processos de respeito, empatia, comunicação, diálogo, procurando compreender não somente as questões de enfermidades, mas também as necessidades biológicas, psicológicas, sociais, culturais, religiosas e valorativas do paciente.

Portanto, é importante que tais profissionais repensem em suas atitudes e ações, assim como, é necessário a garantia das políticas públicas para satisfazer as recomendações especificas de prioridade as pessoas idosas, com vista a garantir o cuidado humanizado e o bem-estar da pessoa idosa, possibilitando um envelhecimento mais tranquilo, saudável e de qualidade.

Destarte, fica claro que os profissionais da enfermagem que atuam na Atenção Básica necessitam de capacitação na área da geriatria e gerontologia; as instituições de saúde precisam de mais estrutura de recursos materiais e humanos; as famílias dos idosos precisam de maiores esclarecimentos e amparo por parte das instituições e dos profissionais de saúde acerca do cuidado a população idosa; os idosos necessitam conhecer mais amplamente sobre seus direitos e; São necessários programas, políticas e ações mais eficientes nos sistemas públicos de atenção à saúde do idoso.

\section{Referências}

Alberti, G. F., Espíndola, R. B., \& Carvalho, S. O. R. M. (2014). Abordagem ao idoso na estratégia de saúde da família e as implicações para a atuação do enfermeiro. J. res.: fundam. care. Online, 6 (2), 695-702.

Bardin, L. (2011). Análise de conteúdo. Edições 70.

Botelho, L. L. R., Cunha, C. C. A., \& Macedo, M. (2011). O método da revisão integrativa nos estudos organizacionais. Gestão Socied, 5 (11), $121-136$.

Brasil, M. S. (2010). Programa Nacional de Humanização da Assistência. Ministério da Saúde, 69 p.

Brito, R. F. S. L. V., et al. (2015). O idoso na estratégia saúde da família: atuação do enfermeiro durante o envelhecimento ativo. R. Interd, 8 (4), $99-108$.

Campos, C. N. A., et al. (2012). Reinventando práticas de enfermagem na educação em saúde: teatro com idosos. Esc Anna Nery (impr.), 16 (3), $588-596$.

Costa, J. B., Furtado, L. G. S., \& Girard, C. C. P. (2019). Saberes e práticas do enfermeiro na consulta com o idoso na estratégia saúde da família. Rev. Aten. Saúde, São Caetano do Sul, 17 (62), 24-30.

Emiliano, M. S., et al. (2017). A percepção da consulta de enfermagem por idosos e seus cuidadores. Rev enferm UFPE on line, 11 (5), $1791-1797$.

Freitas, E. V. (2017). Tratado de geriatria e gerontologia. (4a ed.), Guanabara Koogan.

Freitas, M. A., \& Alvarez, A. M. (2020). Melhores práticas de enfermagem na saúde da pessoa idosa. Rev enferm UFPE, 14 (244049), 1-11.

Instituto Brasileiro de Geografia e Estatística (2018). Projeções da população: Brasil e unidades da federação: revisão 2018. IBGE, Coordenação de População e Indicadores Sociais. (2a ed.), IBGE.

Leite, B. S., et al. (2016). Consultas de enfermagem aos idosos em assistência básica no intercâmbio estudantil internacional: relato de experiência. Rev enferm UFPE on line., 10 (4), 3710-3715.

Linck, C. L., \& Crossetti, M. G. O. (2011). Fragilidade no idoso: o que vem sendo produzido pela enfermagem. Rev Gaúcha Enferm., 32 (2), $385-393$.

Menezes, T. M. O., et al. (2020). Acolhimento e cuidado da enfermeira na estratégia saúde da família: percepções da pessoa idosa. REME - Rev Min Enferm, 24 (1304), 1-8.

Moreira, V. G. (2017). Biologia do envelhecimento. In Freitas, EV e Py, L. Tratado de geriatria e gerontologia. (4a ed.), Guanabara Koogan.

Nogueira, I. S., et al. (2016). Intervenção domiciliar como ferramenta para o cuidado de enfermagem: avaliação da satisfação de idosos. Rev Gaúcha Enferm., 37 (esp), 1-7.

Nunes, J. D., et al. (2017). Indicadores de incapacidade funcional e fatores associados em idosos: estudo de base populacional em Bagé, Rio Grande do Sul. Epidemiol. Serv. Saude, 26 (2), 295-304.

Oliveira, A. M. S., \& Menezes, T. M. O. (2014). A enfermeira no cuidado ao idoso na estratégia saúde da família: sentidos do vivido. Rev enferm UERJ, 22 (4), 513-518.

Oliveira, J. M. M., et al. (2013). O cuidado de enfermagem na visita domiciliar gerontológica: uma perspectiva humanística. Cienc Cuid Saude., 12 (1), 170176.

Pompeo, D. A., Rossi, L. A., \& Galvao, C. M. (2009). Revisão integrativa: etapa inicial do processo de validação de diagnóstico de enfermagem. Acta paul. enferm. 22 (4), 434-438. 
Research, Society and Development, v. 10, n. 10, e395101019005, 2021

(CC BY 4.0) | ISSN 2525-3409 | DOI: http://dx.doi.org/10.33448/rsd-v10i10.19005

Resende, J. O., et al. (2015). Assistência do enfermeiro ao idoso na estratégia saúde da família. R. Enferm. Cent. O. Min., 5 (3), 1831 -1843.

Rocha, F. C. V., et al. (2011). O cuidado do enfermeiro ao idoso na estratégia saúde da família. Rev. enferm. UERJ, Rio de Janeiro, 19 (2), $186-191$.

Sampaio, S. N., et al. (2018). Visão da pessoa idosa sobre o atendimento do enfermeiro da Atenção Básica. Rev baiana enferm., 3 (2), 1-9.

Santos, R. S., et al. (2013). A assistência domiciliar ao idoso na perspectiva dos enfermeiros. Rev enferm UFPE on line, 7 (1), 697-705.

Silva, P. L. N., et al. (2014). Assistência psicológica e de enfermagem ao idoso na atenção primária. J. res.: fundam. care. online., 6 (4), $1707-1718$.

Silva, A. A., \& Borges, M. M. M. C. (2014). Humanização da Assistência de Enfermagem ao idoso em uma Unidade de Saúde da Família. Rev. Enf. Integrada., 1 (1), 11-24.

Silva, H. P., \& Silva, J. L. S. (2017). Humanização da assistência de enfermagem ao idoso. 2017. 31f. Trabalho de Conclusão de Curso. (Bacharel em Enfermagem). Faculdade do Instituto Brasil (FIBRA). Instituto Brasil de Ciência \& Tecnologia LTDA.

Silva, J. P. A. (2019). Humanização e cuidados de enfermagem à saúde da pessoa idosa. 2019. 27f. Trabalho de Conclusão de Curso - TCC (Bacharelado em Enfermagem). Centro Universitário do Planalto Central Apparecido dos Santos - Uniceplac. Gama-DF.

Sousa, L., \& Ribeiro, A. P. (2013). Prestar cuidados de enfermagem a pessoas idosas: experiências e impactos. Saúde Soc., 22 (3), 866-877.

Tavares, R. E., et al. (2017). Envelhecimento saudável na perspectiva de idosos: uma revisão integrativa. Rev. Bras. Geriatr. Gerontol., 20 (6), 889-900.

Ursi, E. S. (2015). Prevenção de lesões de pele no perioperatório: revisão integrativa da literatura. [dissertação]. Ribeirão Preto: Universidade de São Paulo, Escola de Enfermagem de Ribeirão Preto.

Veras, R. P., \& Oliveira, M. (2018). Envelhecer no Brasil: a construção de um modelo de cuidado. Ciência \& Saúde Coletiva, 23 (6), $1929-1936$.

Vieira, P. F., \& Almeida, M. A. R. (2020). Humanização da assistência de enfermagem em pacientes idosos. Rev Inic Cient Ext., 3 (1), $371-378$.

Zoboli, E. L. C. P., \& Schveitzer, M. C. (2013). Valores de enfermagem como prática social: uma meta-síntese qualitativa. Rev. Latino-am. Enfermagem., 21 (3), 695-703. 